\title{
Prioritizing risks of antibiotic resistance genes in all metagenomes
}

\author{
José L. Martínez, Teresa M. Coque and Fernando Baquero
}

We have read with interest the Correspondence on our Opinion article (What is a resistance gene? Ranking risks in resistomes. Nature Rev. Microbiol. 13, 116-123 (2015) $)^{1}$ by Bengtsson-Palme and Larsson (Antibiotic resistance genes in the environment: prioritizing risks Nature Rev. Microbiol. http://dx.doi.org/10.1038/ nrmicro3399-c1 $)^{2}$ and appreciate the observations raised by the authors. As they acknowledge, our article proposes the first framework (termed resistance readiness condition (RESCon)) for evaluating the risks associated with the presence of antibiotic resistance genes in bacterial metagenomes. Here, we wish to clarify and refine some of the arguments that this framework is based on.

The framework we propose relies on two fundamental principles: the likelihood of a known antibiotic resistance gene having a harmful effect on human health (during antibiotic therapy) and whether or not it is likely to transfer to human pathogens (the probability of which increases if it is located on a mobile genetic element). Following this scheme, we consider those resistance genes that are present on mobile elements and for which a role in resistance in human pathogens has been demonstrated to represent the highest risk (defined as RESCon 1). Novel resistance genes are ascribed a lower risk category in our framework if there is no evidence that they have a detrimental influence on the outcome of antibiotic therapy (RESCon 3), whereas the risk is scored higher (RESCon 2) if they are associated with mobile elements. In their Correspondence, Bengtsson-Palme and Larsson suggest that risk order should be reversed in our scheme, such that novel resistance genes present a higher risk because known resistance determinants are likely to be widespread and thus, their propensity to transfer to a pathogen would be minimal. Although this seems like a reasonable argument, in our view, current knowledge of the ecology of antibiotic resistance does not support a change in our risk-assessment scheme.

First, our framework does not only consider environmental ecosystems, as the ranking system also applies to resistance genes in human and animal microbiomes, in which transfer of resistance genes among pathogens and commensals is likely to occur ${ }^{2,3}$. In the case of genes that are present in the microbiomes of animals, the risk may be quite high as the transfer of genes via mobile genetic elements has been documented between human and animal bacteria ${ }^{4}$.

Second, the authors propose that horizontal gene transfer (HGT) of resistance genes among pathogens is rarer in environmental ecosystems than in humans. However, polybacterial infections are not frequent $^{5}$, and the acquisition of resistance genes during infection has been described in just a few cases ${ }^{6,7}$. By contrast, in nonclinical settings, such as wastewater treatment plants, pathogenic and non-pathogenic bacteria are present at high densities and the likelihood of HGT of resistance genes is expected to be high, particularly if the waste contains antibiotics that provide selective pressure for the maintenance of resistance ${ }^{8}$. In this scenario, although the first step in the acquisition of a resistance gene is likely to occur in a non-clinical setting, with the donor and recipient being environmental strains ${ }^{9}$, crucial secondary transfer events can subsequently occur in these ecosystems that may result in the transfer of the resistance gene to pathogens.

Third, Bengtsson-Palme and Larsson focus on the risks for gene transfer to a pathogen but, to clarify, the framework we propose concerns the risks for human health that are associated with the detection of these genes in metagenomic datasets, not just the transfer of these genes. For example, the presence of bla ${ }_{\mathrm{NDM} 1}$ (which encodes New Delhi metallo$\beta$-lactamase) in drinking water distribution networks equates to a risk for human health, irrespective of whether the gene is transferred to a novel host in this habitat ${ }^{10}$.

Fourth, the detection of a novel resistance gene does not necessarily mean that it will be transferred to a human pathogen. The principles used to predict the emergence of resistance distinguish between the potential for transfer and whether transfer actually occurs, which is an important distinction as only the latter scenario would be considered a risk for human health ${ }^{11}$. Transfer requires the connectivity of bacterial populations ${ }^{12}$, and the probability of transmission and subsequent fixation depends on the bottlenecks discussed in our Opinion article ${ }^{1}$. There is a lack of robust tools to predict which gene among the several thousand characterized genes in bacterial communities ${ }^{13}$ - including relevant antibiotic resistance genes (such as those encoding carbapenemases $)^{14}-$ will transfer to a pathogen. Considering this large degree of unpredictability ${ }^{15}$, we believe resistance genes that are known to constitute a health risk should be ranked higher than novel resistance genes in our scheme. Nevertheless, we agree that novel resistance genes are also relevant, and as such they are ranked immediately below those genes already known to constitute a health risk.

We also agree that identifying hotspots for the emergence and dissemination of resistance is highly relevant ${ }^{15-18}$, and environments containing waste from antibiotic production facilities are chief among these, particularly if these sites also harbour potential recipients for transfer. Studying the correlations between antibiotic resistance, the presence of antibiotics and the composition of microbial populations might help in identifying these hotspots. These analyses should distinguish between genes that are present on mobile genetic elements and intrinsic genes, which are simply markers of the presence of a given microorganism in an ecosystem and not necessarily a risk for human health.

All new classification schemes require the input of the community. As such, we thank Bengtsson-Palme and Larsson for their observations, which have raised interesting discussion points that we believe have clarified our proposed framework.

José L. Martinez is at the Departamento de Biotecnología Microbiana, Centro Nacional de Biotecnología, Consejo Superior de Investigaciones Científicas (CSIC), Darwin 3, Cantoblanco, Madrid Spain; and the Unidad de Resistencia a Antibióticos y Virulencia Bacteriana asociada al CSIC, Madrid, Spain.

Teresa M. Coque and Fernando Baquero are at the
Unidad de Resistencia a Antibióticos y Virulencia
Bacteriana asociada al Consejo Superior de
Investigaciones Científicas (CSIC), Madrid, Spain;
and the Departamento de Microbiología,
Hospital Universitario Ramón y Cajal, Instituto
Ramón y Cajal de Investigación Sanitaria (IRYCIS), Madrid 28034, Spain.

Correspondence to J.L.M. e-mail:jlmtnez@cnb.csic.es doi: 10.1038/nrmicro3399-c2 Published online 27 April 2015

1. Martínez, J. L., Coque, T. M. \& Baquero, F. What is a resistance gene? Ranking risk in resistomes. Nature Rev. Microbiol. 13, 116-123 (2015). 
CORRESPONDENCE

2. Bengtsson-Palme, J. \& Larsson, J. Antibiotic resistance genes in the environment: prioritizing risks. Nature Rev. Microbiol. http://dx.doi.org/10.1038/ nrmicro3399-c1 (2015).

3. Salyers, A. A., Gupta, A. \& Wang, Y. Human intestinal bacteria as reservoirs for antibiotic resistance genes. Trends Microbiol. 12, 412-416 (2004).

4. de Been, M. et al. Dissemination of cephalosporin resistance genes between Escherichia coli strains from farm animals and humans by specific plasmid lineages. PLoS Genet. 10, e1004776 (2014).

5. Wisplinghoff, H. et al. Nosocomial bloodstream infections in US hospitals: analysis of 24,179 cases from a prospective nationwide surveillance study. Clin. Infect. Dis. 39, 309-317 (2004)

6. Su, L. H. et al. In vivo acquisition of ceftriaxone resistance in Salmonella enterica serotype anatum. Antimicrob. Agents Chemother. 47, 563-567 (2003).

7. Martínez-Suárez, J. V. et al. Acquisition of antibiotic resistance plasmids in vivo by extraintestinal Salmonella spp. J. Antimicrob. Chemother. 20 , 452-453 (1987)

8. Baquero, F., Martínez, J. L. \& Canton, R. Antibiotics and antibiotic resistance in water environments. Curr. Opin. Biotechnol. 19, 260-265 (2008).

9. Martínez, J. L. Antibiotics and antibiotic resistance genes in natural environments. Science 321, 365-367 (2008).

10. Walsh, T. R., Weeks, J., Livermore, D. M. $\&$ Toleman, M. A. Dissemination of NDM-1 positive bacteria in the New Delhi environment and its implications for human health: an environmental point prevalence study. Lancet Infect. Dis. 11, 355-362 (2011).

11. Martínez, J. L., Baquero, F. \& Andersson, D. I. Predicting antibiotic resistance. Nature Rev. Microbiol. 5, 958-965 (2007)

12. Popa, O. \& Dagan, T. Trends and barriers to lateral gene transfer in prokaryotes. Curr. Opin. Microbiol. 14, 615-623 (2011)

13. Forsberg, K. J. et al. Bacterial phylogeny structures soil resistomes across habitats. Nature 509, 61 2-616 (2014)

14. EFSA-BIOHAZ-Panel. Scientific opinion on carbapenem resistance in food animal ecosystems EFSA J. 11, 3501 (2013)

15. Martínez, J. L. et al. A global view of antibiotic resistance. FEMS Microbiol. Rev. 33, 44-65 (2009).

16. Baquero, F., Alvarez-Ortega, C. \& Martínez, J. L. Ecology and evolution of antibiotic resistance. Environ. Microbiol. Rep. 1, 469-476 (2009)

17. Martinez, J. L. The role of natural environments in the evolution of resistance traits in pathogenic bacteria. Proc. Biol. Sci. 276, 2521-2530 (2009).

18. Martínez, J. L. Environmental pollution by antibiotics and by antibiotic resistance determinants. Environ. Pollut. 157, 2893-2902 (2009).

Competing interests statement

The authors declare no competing interests. 